\title{
SLC34A2 Up-regulation And SLC4A4 Down-regulation Correlates With Invasion, Metastasis, And The MAPK Signaling Pathway In Papillary Thyroid Carcinomas
}

\author{
Fengyan Huang1, Haitao Wang2, Juan Xiao ${ }^{3}$, Chunchun Shao ${ }^{3}$, Yong Zhou', Wei Cong5, Maosong Gong5, \\ Jingfu Sun ${ }^{5}$, Liqun Shan 5 , Zhanyu Hao ${ }^{5}$, Lihua Wang ${ }^{1}$, Shouluan Ding 3 , Zhigang $\mathrm{Yu}^{6}$, Jianing Liu, \\ Hongying Jia $1,3 \bowtie$
}

1. Department of Epidemiology and Health Statistics, School of Public Health, Cheeloo College of Medicine, Shandong University, Jinan, Shandong 250012, P.R. China

2. Department of pathology, The Second Hospital, Cheeloo College of Medicine, Shandong University, Jinan, Shandong 250033, P.R. China

3. Evidence based medicine, The Second Hospital, Cheeloo College of Medicine, Shandong University, Jinan, Shandong 250033, P.R. China

4. Medical laboratory center, The Second Hospital, Cheeloo College of Medicine, Shandong University, Jinan, Shandong 250033, P.R. China

5. Department of Thyroid Surgery, The Second Hospital, Cheeloo College of Medicine, Shandong University, Jinan, Shandong 250033, P.R. China

6. Department of Breast Surgery, The Second Hospital, Cheeloo College of Medicine, Shandong University, Jinan, Shandong 250033, P.R. China

$\triangle$ Corresponding author: Zhigang Yu, Department of Breast Surgery, The Second Hospital, Cheeloo College of Medicine, Shandong University, 247 Beiyuan Dajie, Jinan, Shandong 250033, PR China, Email address: yuzhigang@sdu.edu.cn; Jianing Liu, Department of Thyroid Surgery, The Second Hospital, Cheeloo College of Medicine, Shandong University, 247 Beiyuan Dajie, Jinan, Shandong 250033, PR China, E-mail address: 15153169328@163.com; Hongying Jia, School of Public Health, Cheeloo College of Medicine, Shandong University, 44 Wenhua Xi Road, Jinan, Shandong 250012, P.R. China; The Second Hospital, Cheeloo College of Medicine, Shandong University, 247 Beiyuan Dajie, Jinan, Shandong 250033, P.R. China. E-mail: jiahongying@sdu.edu.cn.

(c) The author(s). This is an open access article distributed under the terms of the Creative Commons Attribution License (https://creativecommons.org/licenses/by/4.0/). See http://ivyspring.com/terms for full terms and conditions.

Received: 2020.12.03; Accepted: 2021.06.28; Published: 2021.07.13

\begin{abstract}
Papillary thyroid carcinoma (PTC) is one of the fastest growing endocrine system malignant carcinomas detected over the past decade. Unfortunately, more than $25 \%$ of PTC patients are characterized by their aggressiveness and subsequent metastasis; these characteristics usually indicate poor prognosis. Recently, increasing evidence has suggested that solute carrier (SLC) transporters may play a pivotal role in the initiation, invasion and metastasis of human carcinoma. However, the expression and clinicopathological significance of SLC transporters in patients with PTC remains undetermined. In this study, we aimed to elucidate how the differential expression of SLC transporters affects clinicopathological features, as well as determine the possible regulatory signaling pathways involved. Three differentially expressed SLC transporters were screened from the Gene Expression Omnibus (GEO) and The Cancer Genome Atlas (TCGA) database using a bioinformatics approach. The results indicated that high SLC34A2 and low SLC4A4 protein expression exhibited a higher percentage of capsular invasion and extra-thyroid metastasis in patients. Logistic regression analysis showed that high SLC34A2 expression in tumors was identified as an independent risk factor for capsular invasion [odds ratio $(\mathrm{OR})=11.400,95 \%$ confidence interval $(\mathrm{Cl})=1.733-74.995, \mathrm{P}=0.011$ ] and extra-thyroid metastasis $(\mathrm{OR}=4.920,95 \% \mathrm{Cl}=1.234-19.623, \mathrm{P}=0.024)$, while low SLC4A4 expression in tumors was only identified as independent risk factors for extra-thyroid metastasis $(\mathrm{OR}=8.568,95 \% \mathrm{Cl}=1.186-61.906$, $\mathrm{P}=0.033$ ). Specifically, for tumors with capsular invasion and extra-thyroid metastasis, the protein expression staining of SLC34A2 was markedly enhanced in the cytoplasm of follicular epithelial cells, contrastingly, SLC4A4 expression was notably weakened in the cytomembrane and nucleus. Intriguingly, both high SLC34A2 and low SLC4A4 protein expression were significantly linked to a high urinary iodine concentration in patients with PTC. Mechanistically, compared with adjacent normal thyroids, P-ERK was significantly up-regulated by $17.8 \%$ in the invading tumor; $\mathrm{p}$-ERK, $\mathrm{p}-\mathrm{JNK}$, and p-P38 were markedly up-regulated by $29.2 \%, 67.1 \%$, and $38.9 \%$ for metastatic tumors, respectively. Importantly, SLC4A4 negatively correlated with $\mathrm{p}-J \mathrm{NK}(\mathrm{r}=-0.696, \mathrm{P}=0.004)$ and $\mathrm{p}-\mathrm{P} 38$ ( $r=-0.534, \mathrm{P}=0.049)$. In conclusion, we suggest that up-regulated SLC34A2 (mainly in the cytoplasm) and down-regulated SLC4A4 (mainly in the cytomembrane and nucleus), which might be attributed to excess iodine intake, were closely linked to extra-thyroid metastasis in PTCs. Furthermore, this effect of SLC4A4 may be through the activation of JNK/P38 MAPK signaling pathway. Future in vivo and in vitro gain- or loss-of-function experiments are needed to verify these findings and further elucidate the deeper molecular mechanisms.
\end{abstract}

Key words: papillary thyroid carcinoma, SLC transporters, invasion, metastasis, MAPK signaling pathway 


\section{Introduction}

Thyroid cancer is the fastest growing endocrine system malignant cancer in recent decades [1]. Papillary thyroid cancer (PTC) is the most predominant of the histopathological types, with $84 \%$ accounting for thyroid cancer [1]. The majority of patients with PTC possess indolent progression. Unfortunately, more than $25 \%$ of patients with PTC are characterized by the disease's aggressiveness and metastatic nature; these characteristics usually indicate a poor prognosis [2, 3]. However, the exploration of clinical biomarkers and molecular mechanisms for the invasion and metastasis of PTCs remains insufficient.

Solute carrier (SLC) transporters represent the largest family of transmembrane proteins encoded in the human genome [4]. These transporters utilize an electrochemical or ion gradient to facilitate the exchange of various substances, including endogenous or xenobiotic compounds as well as molecules across the biological membranes, such as sugars, nucleotides, amino acids, nutrients, and drugs $[5,6]$. Recently, a several studies have highlighted that SLC transporters might play a critical role in the initiation, progression, metastasis, and prognosis of human carcinoma [7, 8]. For instance, Kikuchi et al [9] found that up-regulated SLC37A1 was significantly associated with the venous invasion and liver metastasis of colorectal cancer. Moreover, Ding et al [10] indicated that SLC39A7, SLC39A11, and SLC39A14 were significantly linked to favorable prognosis in patients with gastric cancer. El-Ansari et al [11] utilized co-operative expression analysis and demonstrated that SLC1A5, SLC7A5, and SLC3A2 were distinctly correlated to both the high proliferation and aggressive nature of the ER+ breast cancer. He et al [12] indicated that SLC34A2 might boost the proliferation, cell cycle progression, migration, invasion, and adhesion of PTC cell in vitro via PTEN/AKT/FOXO3 signaling pathway. Despite all aforementioned findings, the effect of the expression and clinicopathological significance of SLC transporters on PTCs has not yet been fully elucidated.

Therefore, in this study, we aimed to evaluate the differential expression of SLC transporters and determine their effect on clinicopathological characteristics, further devoted to reveal possible regulatory signaling pathways involved. Undoubtedly, the results will provide a novel insight into expound the deeper mechanism of invasion and metastasis of patients with PTC, and offer a research reference of clinical biomarkers and new therapeutic targets to some extent.

\section{Materials and Methods}

\section{Study population selected}

This study involved patients with thyroid nodules who underwent thyroidectomy at the Department of Thyroid Surgery, The Second Hospital of Shandong University (Jinan, China), from July 2019 to December 2019. The included criteria were as follows: i) patients with initial thyroidectomy; ii) patients were diagnosed as the resectable primary tumors; iii) patients hadn't received previous treatment for the tumor before the surgical excision; iv) recently hadn't received the therapeutic iodine (2 months) nor used of anti-thyroid drugs or thyroid hormone therapy; v) patients hadn't accompanied with kidney or liver dysfunction, or other systemic diseases. After excluding medullary, follicular, and undifferentiated thyroid carcinoma, 48 cases of PTC remained.

The pathohistological type was determined by the pathologist from the Department of Pathology of The Second Hospital of Shandong University. Pathological characteristics, including primary tumor size and location, capsular invasion, extra-thyroid metastasis, complication, and lymph node location [which were evaluated based on the National Comprehensive Carcinoma Network guidelines for thyroid carcinoma recommendation (https:// www.nccn.org)], were extracted from the pathological report; particularly, after routine pathological diagnoses, lymph nodes were detected in 43 cases of PTC among 48 patients, and other 5 cases cannot found any lymph nodes. Refer to previously published standards [13], patients with thyroid nodules were divided into two groups in present study according to Thyroid Imaging Reporting and Data System (TIRADS) grading: TIRADS 2, 3, 4a, and $4 \mathrm{~b}$ were in a group, TIRADS $4 \mathrm{c}$ and 5 were in a group. Descriptions of nodule feathers was obtained from the latest ultrasound reports.

This study was reviewed and approved by The Research Ethics committee of The Second Hospital of Shandong University [no. KYLL-2019(KJ)P-0084]. This study was performed in accordance with the ethical standards of the Declaration of Helsinki. Written informed consent was obtained from all patients before using their samples and clinical data.

\section{Bioinformatics analysis}

The mRNA expression profiles of GSE3678, GSE29265, GSE33630, and GSE50901 microarrays in tumor tissues and matched normal thyroid tissues were compared using the Gene Expression Omnibus (http://www.ncbi.nlm.nih.gov/geo/) database (GEO). The screening process is shown in 
Supplementary Table 1 . The raw data were downloaded and analyzed using the "limma" package in $\mathrm{R}$ software (version 3.5.1), in which the differentially expressed genes (DEGs) that met the cut-off criteria of an adjusted $\mathrm{P}<0.05$ and a $\mid \log 2$ Fold-change| of $>1.0$ were screened. The package "heatmap.2" was used to perform the hierarchical clustering analysis. The screened DEGs were processed with Venn analysis, and intersection genes were considered as overlapping DEGs. The gene expression profiles were based on RNA-seq and relevant clinical data from The Cancer Genome Atlas (TCGA) database. This comprised 512 PTC tumor tissues and 337 normal thyroid tissues, which were downloaded from the cBioPortal for Cancer Genomics (https://www.cbioportal.org/) online platform [14] and used for expression comparison and survival [including disease/progression-free survival (DFS/PFS)] analysis.

\section{Tissue RNA isolation and real-time PCR (RT-PCR) assay}

Total RNA was extracted from fresh frozen specimens using a RNA reagent kit (No. G3013, Servicebio, China) following the manufacturer's instructions. cDNA was obtained using the RT Synthesis reagent Kit (No. G3330, Servicebio, China) according to manufacturer's instructions. RT-PCR was performed with the LightCycler 480 System (Roche Diagnostics, IN, USA). The PCR profile was as follows: the thermocycling conditions were $95^{\circ} \mathrm{C}$ for $10 \mathrm{~min}$ followed by 40 cycles of $95^{\circ} \mathrm{C}$ for $15 \mathrm{~s}$ and $60^{\circ} \mathrm{C}$ for $60 \mathrm{~s}$. GAPDH was used as a reference gene. The following forward and reverse primers were used for PCR:

SLC34A2, 5'-AGGGCTTATCCACTCACGCT G-3' and 5'-GATGATGAAGACGACGGGAAC-3'; SLC4A4, 5'-AGCCAACAAGTCCAAACCGA-3' and 5'-CTACATACCACGGAAGAGCCAT-3'; SLC25A15, 5'-GCTGCCTGAAGACTTACTCCCA-3' and 5'-TTC TGGCTCTTGGCTATCTTCC-3'; GAPDH, 5'-GGAAG CTTGTCATCAATGGAAATC- $3^{\prime}$ and $5^{\prime}$-TGATGAC CCTTTTGGCTCCC-3'. The relative changes in the gene expression were calculated according to $2-\Delta \Delta \mathrm{Ct}$ method for quantification [15].

\section{Tissue protein extraction and western blotting analysis}

The fresh frozen tissues were washed 3 times in PBS and homogenized ice-cold RIPA buffer containing phosphatase inhibitors, phenylmethanesulfonyl fluoride (PMSF), and protease inhibitor cocktail. Protein concentrations were determined by BCA protein quantification kit [16]. Proteins were separated by $10 \%$ SDS-PAGE with a $4 \%$ stacking gel and transferred to a PVDF membrane. The membranes were blocked with $5 \%$ skim milk for $1.5 \mathrm{~h}$, followed by incubation overnight at $4^{\circ} \mathrm{C}$ with primary antibodies against SLC34A2 (dilution 1:1000; D6W2G, CST, US), SLC4A4 (dilution 1:1000; ab187511, Abcam, UK), SLC25A5 (dilution 1:1000; ab228604, Abcam, UK), ERK (dilution 1:500; bsm-33337M, Bioss, China), p-ERK (dilution 1:500; bs-1522R, Bioss, China), JNK (dilution 1:500; E-AB-60070, Elabscience, China), p-JNK (dilution 1:500; BM4380, Boster, China), P38 (dilution 1:500; E-AB-32459, Elabscience, China), p-P38 (dilution 1:500; E-AB-21027, Elabscience, China), $\beta$-actin (dilution 1:2000; 17AV0412, Zhongshan Golden Bridge, China). After washing 3 times with TBST (TBS containing $0.1 \%$ Tween 20), the membrane was incubated with horseradish peroxidase-conjugated secondary antibody (dilution 1:5000; ZB-2301, Zhongshan Golden Bridge, China). The signal was detected using chemiluminescence reagent (Millipore, cat no. WBKLSO100, Burlington, USA), and the intensity of the integrated optical density of the bands was quantitated using AlphaView SA software. Refer to the published reference standard of the protein expression assessment [3, 11, 12], the quantitative analysis enabled the calculation of the ratio of protein expression in tumor tissue compared to adjacent normal thyroid tissue. If the ratio is $>1.00$, it was defined as high expression; if the ratio is $<1.00$, it was defined as low expression; if there was no significant differences in the expression between the tumor and normal thyroid tissues, it was defined as unchanged.

\section{Immunohistochemical (IHC) staining}

The protein expression localization were performed by using the method of IHC as described in our previously studies $[17,18]$. In brief, the tissues were fixed in $4 \%$ paraformaldehyde and embedded in paraffin. The slides were deparaffinized, rehydrated, and treated with 3\% hydrogen peroxide for $20 \mathrm{~min}$ to inhibit the endogenous peroxidase. The sections were rinsed with distilled water and saturated in phosphate buffered saline for $5 \mathrm{~min}$ and then incubated with a 1:10000 dilution of rabbit anti-polyclonal primary antibody overnight at $4{ }^{\circ} \mathrm{C}$. The staining was visualized using DAB solution and counterstained with hematoxylin. The IHC staining was conducted in accordance with the manufacturer's instructions.

\section{lodine nutrition status evaluation}

The methods of urinary iodine concentration (UIC) detection and quality controls, as well as the water iodine assessment were the same as our previously study [19]. According to the 
recommendations of the World Health Organization, the iodine nutritional status of individual's was categorized into three degrees as follows: i) low UIC $(<100 \mathrm{ug} / \mathrm{l})$, iodine-deficient; ii) adaptive UIC (100-299 ug/l), iodine-adequate; and iii) high UIC $(\geq 300 \mathrm{ug} / \mathrm{l})$, iodine-excessive. The water iodine concentrations for shallow groundwater of domicile residential district of Shandong province were consulted. According to the grading standard for water iodine concentration, Shandong was divided into three groups as follows: i) historically iodine-deficient regions (median water iodine concentration $<10 \mathrm{ug} / \mathrm{l}) ; \quad$ ii) historically iodine-adaptive regions (median water iodine concentration 10-150 ug/1); and iii) historically iodine-excessive regions (median water iodine concentration $>150 \mathrm{ug} / \mathrm{l}$ ).

\section{Statistical analysis}

Data were entered into the software by two researchers and double cross-checked using EpiData 3.0 (https://www.epidata.dk) for quality control. Descriptions of the basic characteristics are expressed as mean \pm standard deviation, or as percentages. For continuous variables, differences between the groups were analyzed using ANOVA, a $t$-test, or a nonparametric analysis of variance test (Kruskal-Wallis $\mathrm{H}$ test and Mann-Whitney U test) in accordance with the concrete types of data. McNemar's test was used for self-matching comparative analyses. Chi-squared or fisher's exact probability method was used to differentiate the rates of different groups. Bonferroni's test was used for multiple comparisons followed by a post-hoc test. Logistic regression analysis was also performed using SPSS. Kaplan-Meier method was conducted to calculate the survival curves. A log-rank test was used to determine differences of the survival rates. Spearman rank test was used for linear correlation analyses. These analyses were all performed by SPSS 22.0 (IBM Corp.) and $\mathrm{R}$ software (version 3.5.1) (https://www.r-project.org). Sigma Plot (version 14.0), GraphPad prism (version 5.0), and Photoshop (version 13.0.1) software were utilized for visualization. The $\mathrm{P}$ value $<0.05$ was regarded as statistically significant.

\section{Results}

\section{Identification of differentially expressed SLC transporters in PTCs}

\section{Bioinformatics analysis using public database}

A total of 1038 DEGs (399 up-regulated and 639 down-regulated) were identified in GSE3678, 1198 DEGs (523 up-regulated and 675 down-regulated) in
GSE29265, 1292 DEGs (658 up-regulated and 634 down-regulated) in GSE50901, and 1790 DEGs (974 up-regulated and 816 down-regulated) in GSE33630 (Figure 1). The visualization analysis of hierarchical clusters demonstrated that the above screened DEGs can notably distinguish tumor tissue from the matched normal thyroid in corresponding microarrays datasets (Figure 2). In total, 270 overlapped DEGs (Supplementary Table 2 and Figure $3 \mathrm{~A})$ were screened from above four microarray datasets. Among these overlapped DEGs, after excluding any non-coding RNA (likes LncRNA SLC26A4-AS1 [20]), remaining SLC transporters were all utilized for expression comparison and survival analysis, using the TCGA clinical database. The results revealed that SLC34A2 was mostly overexpressed in tumors when compared with the normal thyroid. Contrastingly, SLC4A4 and SLC25A15 were mostly decreased in tumors when compared with normal thyroids (Figure 3B-D). Furthermore, the higher expression of SLC34A2 and the lower expression of both SLC4A4 and SLC25A15 were all significantly correlated with shorter DFS/PFS (log-rank test $\mathrm{P}$ value all $<0.05$ ) of patients of PTC (Figure 3E-G). In other words, SLC34A2 usually acts as factor in unfavorable prognoses, whereas SLC4A4 and SLC25A15 act as the preventive factors and can prolong the progress-free periods of PTCs. Thus, these three genes were all chosen for further analysis.

Expression of SLC transporters in both tumor and adjacent normal thyroid

To verify the accuracy of the result of the GEO and TCGA database, we utilized RT-PCR experiment to evaluate SLC34A2, SLC4A4, and SLC25A15 expressions in eight cases of pair matched tissue samples. RT-PCR results showed that expression of SLC34A2 mRNA was drastically up-regulated while SLC4A4 mRNA was drastically down-regulated in tumor tissue, when compared with the adjacent normal thyroid tissue $(\mathrm{P}$ all $<0.05)$. A similar trend was observed at the protein expression level for both SLC34A2 and SLC4A4, as evidenced by western blotting. Unfortunately, both the mRNA and protein expression levels of SLC25A15 was not remarkably different between tumor and adjacent normal thyroid (Figure 4). Thus, SLC34A2 and SLC4A4 were selected for subsequent analysis.

\section{Clinicopathological significance of SLC transporters in patients with PTC}

\section{Comparison analysis}

To explore the possible association between the protein expression of SLC34A2 and SLC4A4 regarding clinicopathological features, western 
blotting was performed on a clinical cross-section consisting of 48 cases of PTCs; including 12 men and 36 women, with a mean age \pm SD of $45.2 \pm 13.4$ years (range 19-68 years). The results of the comparison analysis are displayed in Tables 1 and Table 2 . Approximately half of the PTCs $(43.8 \%, 21 / 48)$ exhibited a high SLC34A2 protein expression in tumor tissue compared with the adjacent normal thyroid tissue; $90.5 \%(19 / 21), 76.2 \%(16 / 21)$, and $76.2 \%(16 / 21)$ of these PTCs with high SLC34A2 exhibited capsular invasion, extra-thyroid metastasis, and high UIC, respectively; the percentage was dramatically higher than that of the unchanged SLC34A2 expression group [54.5\% (12/22), 40.9\% $(9 / 22)$, and $31.8 \%(7 / 22), \mathrm{P}$ all < 0.05]. Almost one-third of PTCs $(33.3 \%, 16 / 48)$ presented a low SLC4A4 protein expression in tumor tissue compared with the adjacent normal thyroid tissue; $93.7 \%$ $(15 / 16), 75.0 \%(12 / 16)$, and $81.2 \%(13 / 16)$ of these PTCs with low SLC4A4 presented capsular invasion, extra-thyroid metastasis, and high UIC, respectively, the percentage was significantly higher than that of the unchanged SLC4A4 expression group [54.2\% $(13 / 24), 33.3 \%(8 / 24)$, and $37.5 \%(9 / 24), \mathrm{P}$ all < 0.05].

\section{Logistic regression analysis}

As shown in Tables 3 and Table 4, logistic regression analysis indicated that PTCs with a high SLC34A2 protein expression in tumor tissue was identified as the independent predictor for the risk of both capsular invasion [univariate analysis: odds ratio $(\mathrm{OR})=7.917,95 \%$ confidence interval $(\mathrm{CI})=1.473-$ 42.538, $\mathrm{P}=0.016$; multivariate analysis: $\mathrm{OR}=11.400$; 95\% CI=1.733-74.995, $\mathrm{P}=0.011)]$ and extra-thyroid metastasis (univariate analysis: $\mathrm{OR}=4.622, \quad 95 \%$ $\mathrm{CI}=1.240-17.266, \quad \mathrm{P}=0.023$; multivariate analysis: $\mathrm{OR}=4.920,95 \% \mathrm{CI}=1.234-19.623, \mathrm{P}=0.024)$ in PTCs. While low SLC4A4 protein expression in tumors (univariate analysis: $\mathrm{OR}=6.000,95 \% \mathrm{CI}=1.458-24.686$, $\mathrm{P}=0.013$; multivariate analysis: $\mathrm{OR}=8.568, \quad 95 \%$ $\mathrm{CI}=1.186-61.906, \mathrm{P}=0.033$ ) was only determined as the independent predictor for the risk of extra-thyroid metastasis of PTCs. This finding indicated that high level of SLC34A2 might be closely associated with the risk of both capsular invasion and extra-thyroid metastasis, while low SLC4A4 was only closely related to the risk of extra-thyroid metastasis.
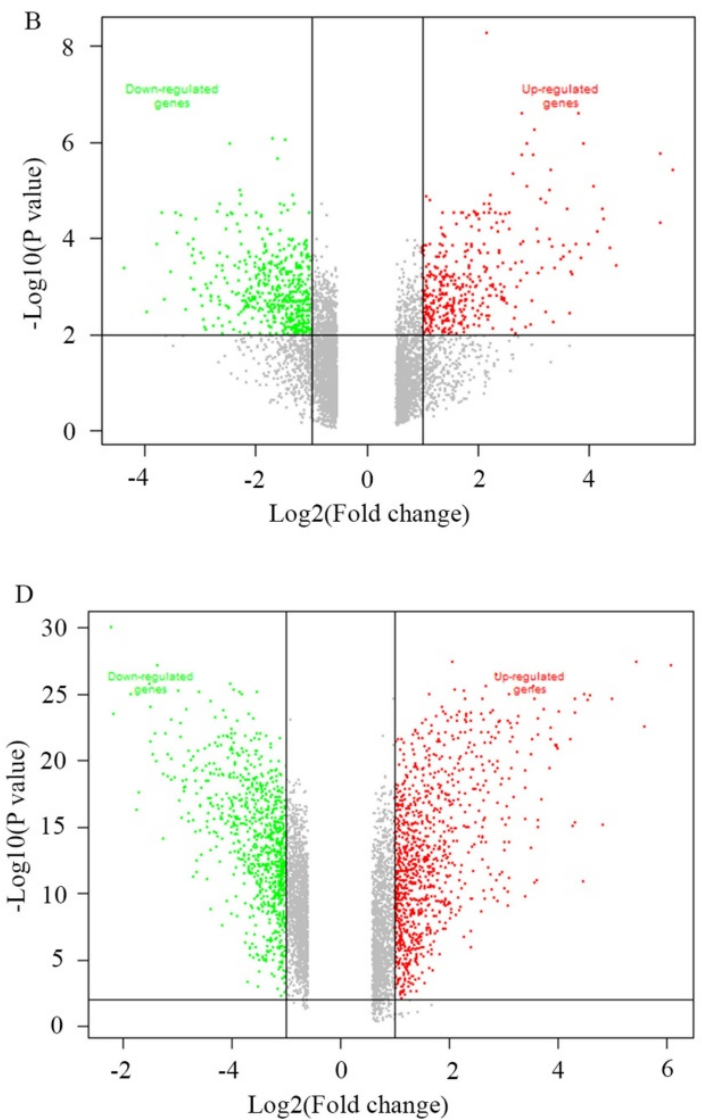

Figure 1. Volcano plot of the genes between tumors with its matched normal thyroids. A-D were namely GSE3678, GSE29265, GSE50901, and GSE33630. P<0.05 and Fold change $>2$. Red spot stands for up-regulated genes in tumors, green spot stands for down-regulated genes in tumors, while gray spot stands for non-difference. 
A

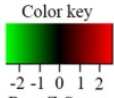

$\begin{array}{ccccccccccc}-2 & -1 & 0 & 1 & 1\end{array}$
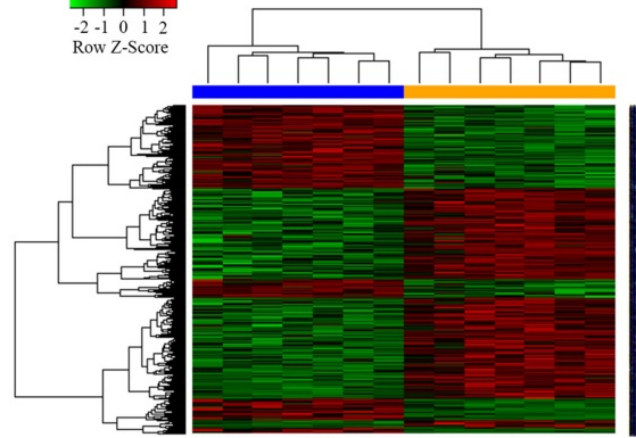

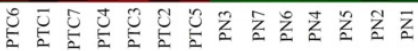
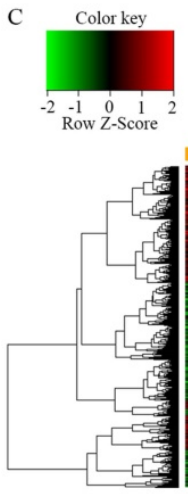
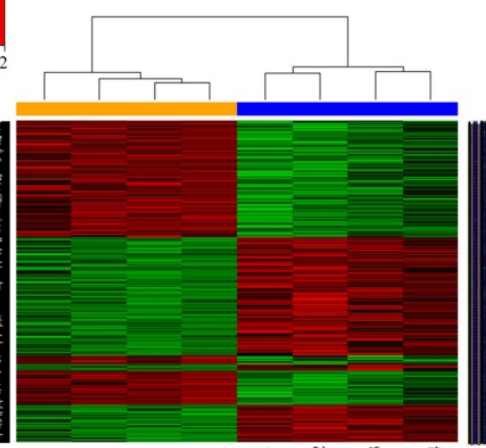

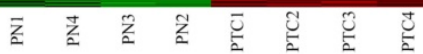

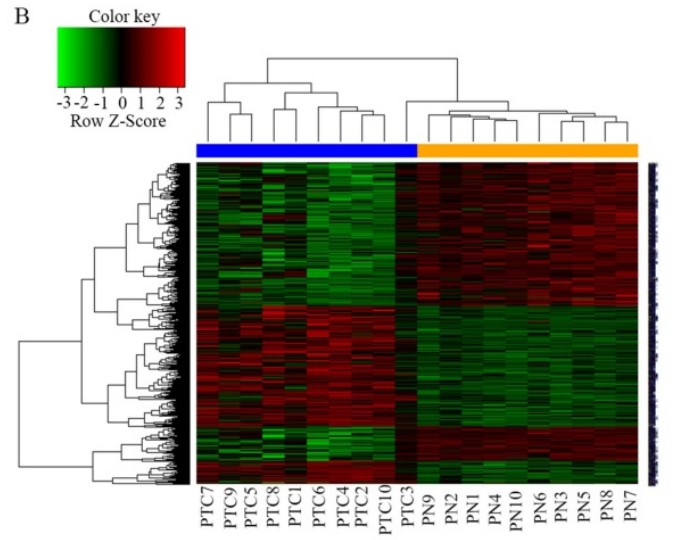

$\mathrm{D}$
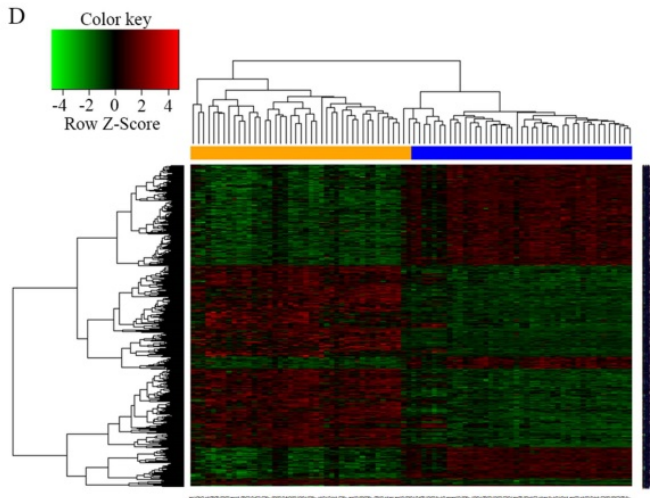

2

Figure 2. Heatmap of the DEGs between tumors with its matched normal thyroid. A-D were namely GSE3678, GSE29265, GSE50901 and GSE33630. P<0.05; Fold change>2. The depth of the color is correlated with genes expression strength on a linear scale, red stands for up-regulated genes, while green stands for down-regulated genes. The genes dendrogram and samples assignment were shown along the left and the bottom. DEGs, differentially expressed genes.
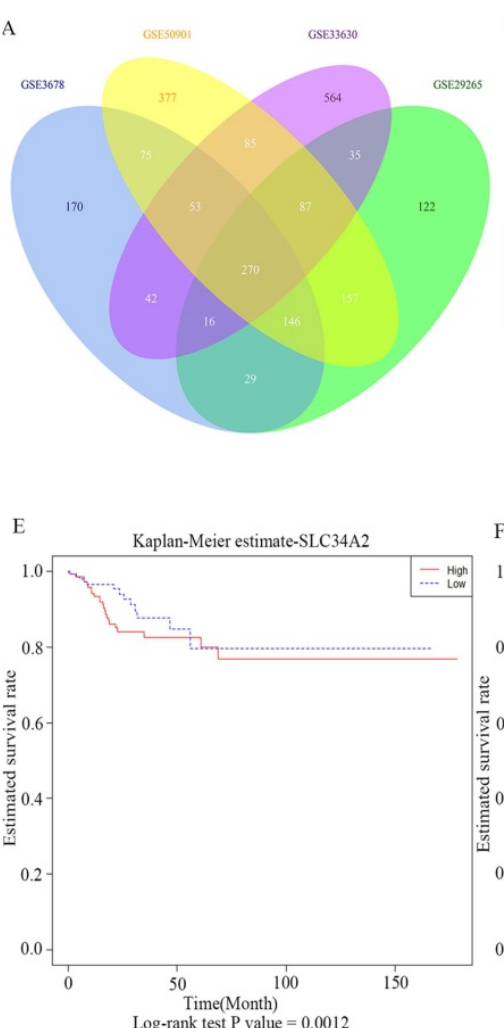

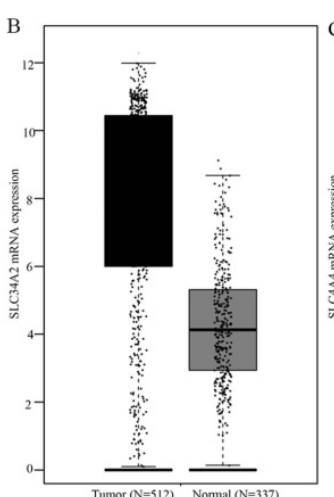

$\mathrm{F}$

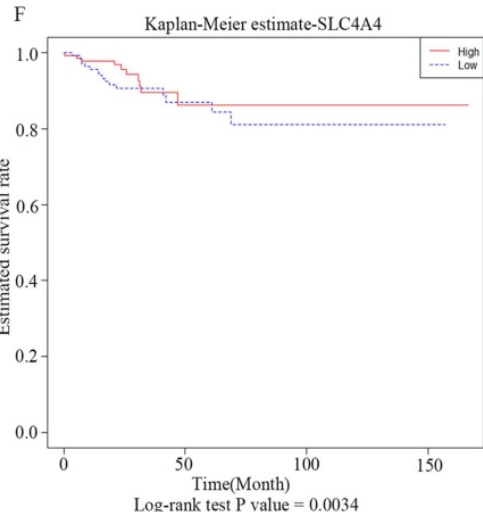

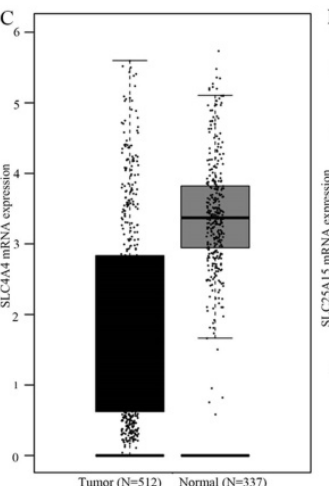
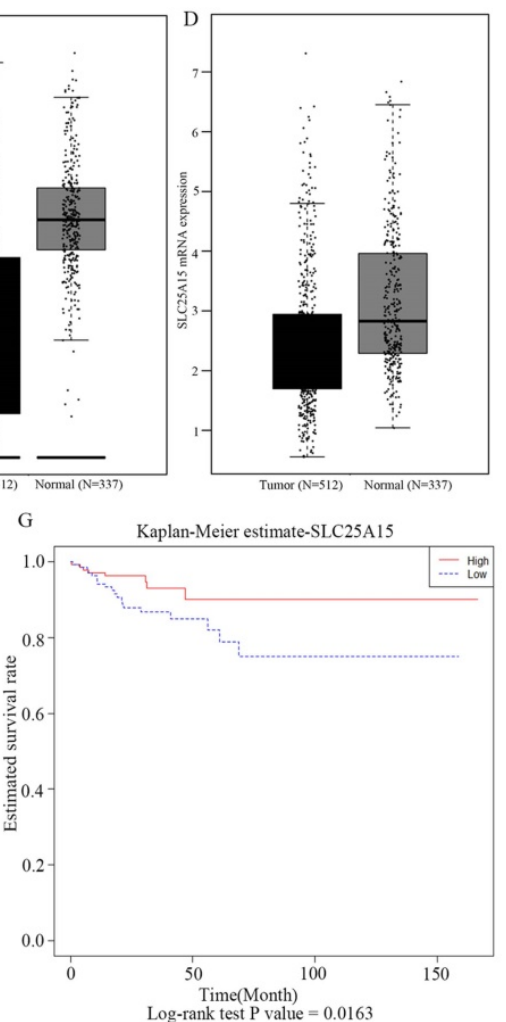

Figure 3. Intersection genes expression and Kaplan-Meier survival analysis based on TCGA clinical cohorts. A was the Venn plot of overlapped DEGs of GSE3678, GSE29265, GSE50901, and GSE33630; B-D were box plot of expression of SLC34A2, SLC4A4, and SLC25A15 in tumor and matched normal thyroid; E-G were the survival curves of SLC34A2, SLC4A4, and SLC25A15, respectively. Black box symbolized the tumor, grey box symbolized the normal. Red line symbolized high expression, blue line symbolized low expression. TCGA: The Cancer Genome Atlas; DEGs: differentially expressed genes. 
Table 1. Association between the expression of SLC34A2 with clinicopathological characteristics of PTC patients (mean $\pm S D, n$, \%)

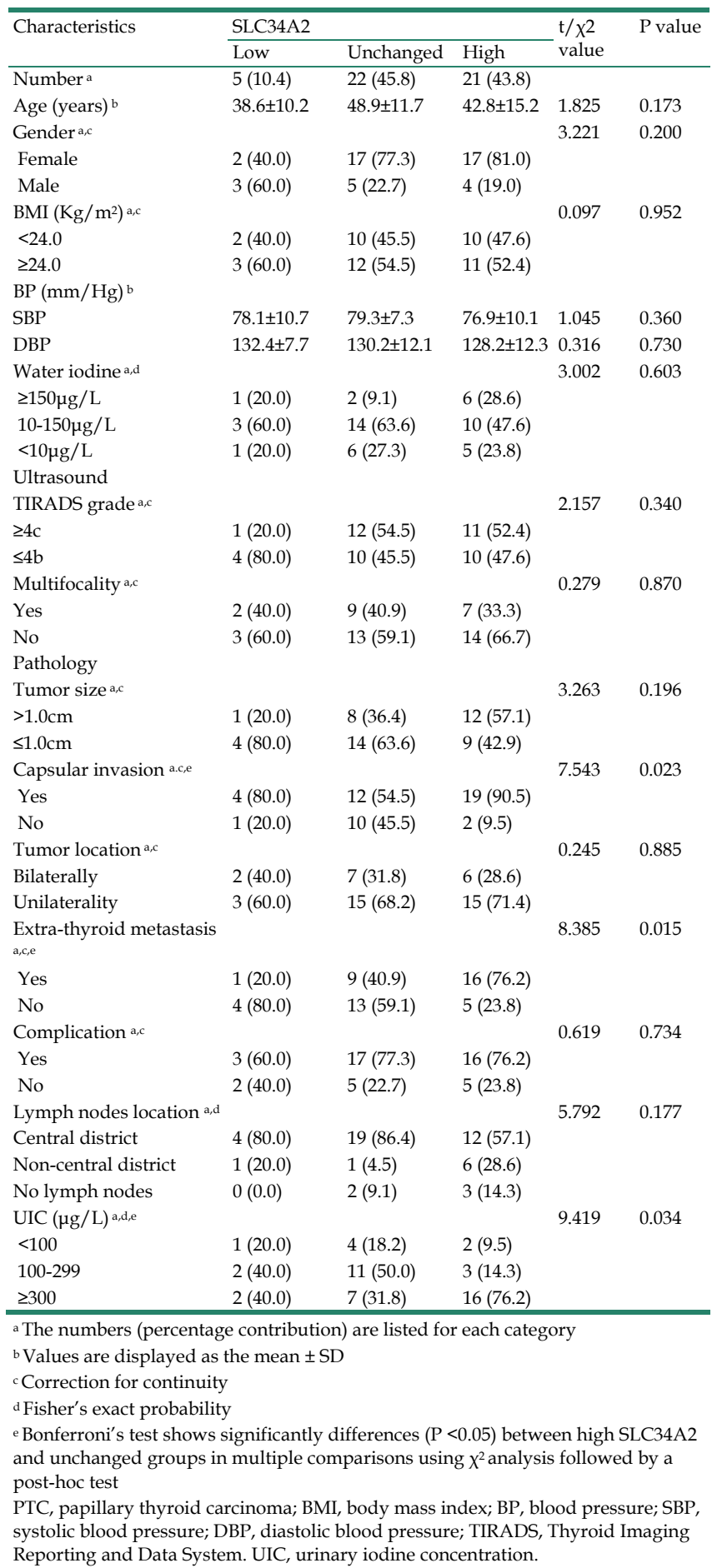

Table 2. Association between the expression of SLC4A4 with clinicopathological characteristics of PTC patients (mean $\pm S D, n$, $\%)$

\begin{tabular}{llllll}
\hline Characteristics & \multicolumn{2}{l}{ SLC4A4 } & & \multicolumn{1}{c}{ t/ $\chi^{2}$} & P value \\
\cline { 2 - 4 } & Low & Unchanged & High & value & \\
\hline Number $^{\mathrm{a}}$ & $16(33.3)$ & $24(50.0)$ & $8(16.7)$ & & \\
Age (years) $^{\mathrm{b}}$ & $38.7 \pm 13.7$ & $48.5 \pm 12.2$ & $47.9 \pm 13.8$ & 2.955 & 0.062 \\
Gender $^{\mathrm{a}, \mathrm{c}}$ & & & & 1.897 & 0.387 \\
\hline
\end{tabular}

\begin{tabular}{|c|c|c|c|c|c|}
\hline \multirow[t]{2}{*}{ Characteristics } & \multicolumn{3}{|l|}{ SLC4A4 } & \multirow{2}{*}{$\begin{array}{l}t / x^{2} \\
\text { value }\end{array}$} & \multirow[t]{2}{*}{$P$ value } \\
\hline & Low & Unchanged & High & & \\
\hline Female & $11(68.7)$ & $20(83.3)$ & $5(62.5)$ & & \\
\hline Male & $5(31.3)$ & $4(16.7)$ & $3(37.5)$ & & \\
\hline BMI $\left(\mathrm{Kg} / \mathrm{m}^{2}\right)^{\mathrm{a}, \mathrm{c}}$ & & & & 0.338 & 0.844 \\
\hline$<24.0$ & $8(50.0)$ & $11(45.8)$ & $3(37.5)$ & & \\
\hline$\geq 24.0$ & $8(50.0)$ & $13(54.2)$ & $5(62.5)$ & & \\
\hline \multicolumn{6}{|l|}{$\mathrm{BP}(\mathrm{mm} / \mathrm{Hg})^{\mathrm{b}}$} \\
\hline SBP & $78.7 \pm 9.1$ & $78.7 \pm 7.5$ & $78.1 \pm 10.7$ & 0.016 & 0.984 \\
\hline DBP & $130.3 \pm 12.3$ & $128.5 \pm 11.6$ & $131.4 \pm 12.1$ & 0.227 & 0.798 \\
\hline Water iodine $\mathrm{a}^{\mathrm{a}, \mathrm{d}}$ & & & & 3.558 & 0.511 \\
\hline$\geq 150 \mu \mathrm{g} / \mathrm{L}$ & $5(31.2)$ & $3(12.5)$ & $1(12.5)$ & & \\
\hline $10-150 \mu \mathrm{g} / \mathrm{L}$ & $8(50.0)$ & $13(54.2)$ & $6(75.0)$ & & \\
\hline$<10 \mu \mathrm{g} / \mathrm{L}$ & $3(18.8)$ & $8(33.3)$ & $1(12.5)$ & & \\
\hline \multicolumn{6}{|l|}{ Ultrasound } \\
\hline TIRADS grade $\mathrm{a}, \mathrm{c}$ & & & & 0.672 & 0.715 \\
\hline$\geq 4 \mathrm{c}$ & $8(50.0)$ & $11(45.8)$ & $5(62.5)$ & & \\
\hline$\leq 4 \mathrm{~b}$ & $8(50.0)$ & $13(54.2)$ & $3(37.5)$ & & \\
\hline Multifocality a,c & & & & 0.790 & 0.674 \\
\hline Yes & $5(31.3)$ & $9(37.5)$ & $4(50.0)$ & & \\
\hline No & $11(68.7)$ & $15(62.5)$ & $4(50.0)$ & & \\
\hline \multicolumn{6}{|l|}{ Pathology } \\
\hline Tumor size $a, c$ & & & & 0.168 & 0.919 \\
\hline$>1.0 \mathrm{~cm}$ & $7(43.7)$ & $10(41.7)$ & $4(50.0)$ & & \\
\hline$\leq 1.0 \mathrm{~cm}$ & $9(56.3)$ & $14(58.3)$ & $4(50.0)$ & & \\
\hline Capsular invasion a,ce & & & & 9.458 & 0.009 \\
\hline Yes & $15(93.7)$ & $13(54.2)$ & $7(87.5)$ & & \\
\hline No & $1(6.3)$ & $11(45.8)$ & $1(12.5)$ & & \\
\hline Tumor location $\mathrm{a}^{\mathrm{a}, \mathrm{c}}$ & & & & 1.842 & 0.398 \\
\hline Bilaterally & $3(18.7)$ & $9(37.5)$ & $3(37.5)$ & & \\
\hline Unilaterality & $13(81.3)$ & $15(62.5)$ & $5(62.5)$ & & \\
\hline $\begin{array}{l}\text { Extra-thyroid metastasis } \\
a, c, e\end{array}$ & & & & 8.664 & 0.013 \\
\hline Yes & $12(75.0)$ & $8(33.3)$ & $6(75.0)$ & & \\
\hline No & $4(25.0)$ & $16(66.7)$ & $2(25.0)$ & & \\
\hline Complication ${ }^{\mathrm{a}, \mathrm{c}}$ & & & & 2.377 & 0.305 \\
\hline Yes & $14(87.5)$ & $16(66.7)$ & $6(75.0)$ & & \\
\hline No & $2(12.5)$ & $8(33.3)$ & $2(25.0)$ & & \\
\hline Lymph nodes location a,d & & & & 1.716 & 0.852 \\
\hline Central district & $11(68.8)$ & $18(75.0)$ & $6(75.0)$ & & \\
\hline Non-central district & $3(18.7)$ & $3(12.5)$ & $2(25.0)$ & & \\
\hline No lymph nodes & $2(12.5)$ & $3(12.5)$ & $0(0.0)$ & & \\
\hline UIC $(\mu g / L) a, d, e$ & & & & 10.713 & 0.019 \\
\hline$<100$ & $2(12.5)$ & $3(12.5)$ & $2(25.0)$ & & \\
\hline $100-299$ & $1(6.3)$ & $12(50.0)$ & $3(37.5)$ & & \\
\hline$\geq 300$ & $13(81.2)$ & $9(37.5)$ & $3(37.5)$ & & \\
\hline \multicolumn{6}{|c|}{ a The numbers (percentage contribution) are listed for each category } \\
\hline \multicolumn{6}{|c|}{${ }^{b}$ Values are displayed as the mean $\pm \mathrm{SD}$} \\
\hline \multicolumn{6}{|c|}{$\begin{array}{l}\text { cCorrection for continuity } \\
\text { d Fisher's exact probability }\end{array}$} \\
\hline dFisher's exact probability & & & & & \\
\hline \multicolumn{6}{|c|}{$\begin{array}{l}\text { e Bonferroni's test shows significantly differences }(\mathrm{P}<0.05) \text { between low SLC4A4 } \\
\text { and unchanged groups in multiple comparisons using } \chi^{2} \text { analysis followed by a } \\
\text { post-hoc test }\end{array}$} \\
\hline $\begin{array}{l}\text { PTC, papillary thyroid ca } \\
\text { systolic blood pressure; }\end{array}$ & arcinoma; BN & , body mass in & ndex; BP, blo & ood press & $\begin{array}{l}\text { ure; SBP, } \\
\text { maging }\end{array}$ \\
\hline
\end{tabular}

Table 3. Logistic regression analysis for predictors of the capsular invasion risk of PTC patients

\begin{tabular}{lllllll}
\hline \multirow{2}{*}{ Expression } & \multicolumn{2}{l}{ Univariate analysis } & \multicolumn{4}{l}{ Multivariate analysis } \\
\cline { 2 - 7 } & OR & $95 \% \mathrm{CI}$ & P value & OR & $95 \% \mathrm{CI}$ & P value \\
\hline SLC34A2 & & & & & & \\
Unchanged & 1.00 & & & & & \\
High & 7.917 & $1.473-42.538$ & 0.016 & 11.400 & $1.733-74.995$ & 0.011 \\
Low & 3.333 & $0.319-34.830$ & 0.315 & 5.417 & $0.280-104.874$ & 0.264 \\
SLC4A4 & & & & 1.00 & & \\
Unchanged & 1.00 & & & 0.360 & $0.015-8.418$ & 0.525 \\
High & 5.923 & $0.628-55.853$ & 0.120 & 7.010 & $0.581-84.557$ & 0.125 \\
Low & 12.692 & $1.438-112.019$ & 0.022 & & & \\
\hline Adjusted potential covariates for multivariate analysis included age, gender, and \\
BMI; PTC, papillary thyroid carcinoma; OR, odds ratio; CI, confidence interval; \\
BMI, body mass index.
\end{tabular}




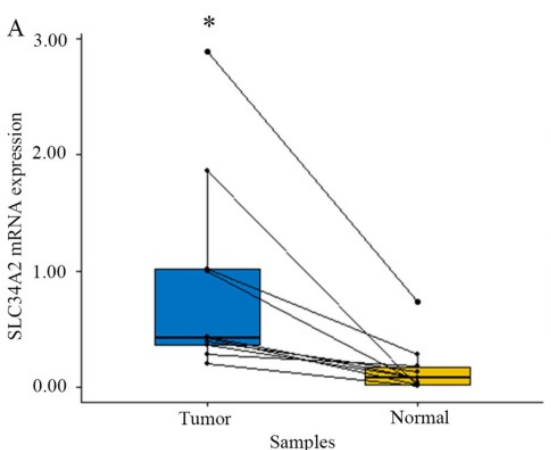

D

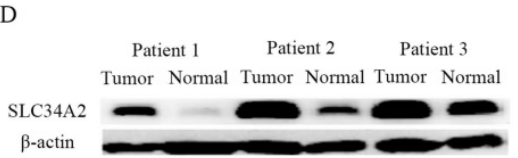

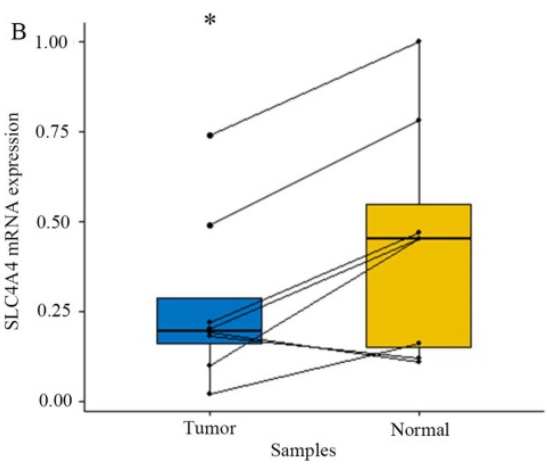

E

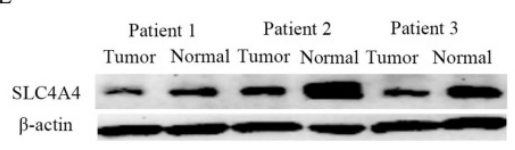

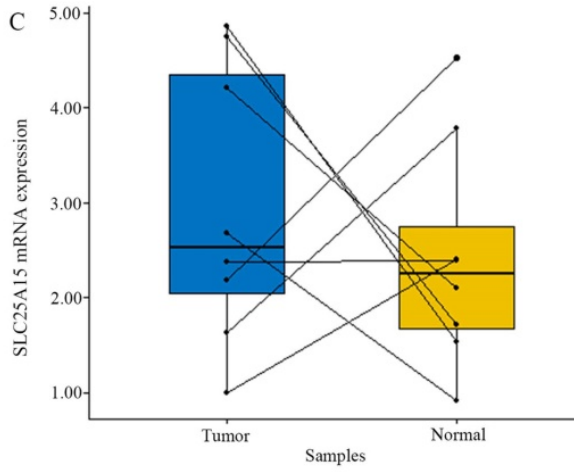

$\mathrm{F}$

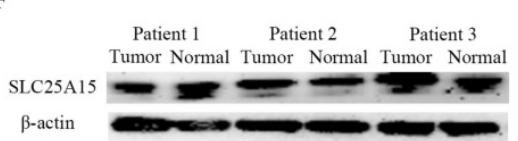

Figure 4. Expression of SLC34A2 ( $A$ and D), SLC4A4 ( $B$ and E), and SLC25A15 ( $C$ and $F$ ) in tumor tissues and adjacent normal thyroid tissues. A-C were the mRNA expression analysis by RT-PCR $(N=8)$; D-F were the representative protein bands of western blotting. “*” represented $P<0.05$.

Table 4. Logistic regression analysis for predictors of the extra-thyroid metastasis risk of PTC patients

\begin{tabular}{lllllll}
\hline \multirow{2}{*}{ Expression } & \multicolumn{2}{l}{ Univariate analysis } & \multicolumn{4}{c}{ Multivariate analysis } \\
\cline { 2 - 7 } & OR & $95 \%$ CI & P value & OR & $95 \%$ CI & P value \\
\hline SLC34A2 & & & & & & \\
Unchanged & 1.00 & & & 1.00 & & \\
High & 4.622 & $1.240-17.226$ & 0.023 & 4.920 & $1.234-19.623$ & 0.024 \\
Low & 0.361 & $0.034-3.788$ & 0.396 & 14.465 & $1.136-184.116$ & 0.040 \\
SLC4A4 & & & & & & \\
Unchanged & 1.00 & & & 1.00 & & \\
High & 6.000 & $0.981-36.715$ & 0.053 & 1.014 & $0.124-8.302$ & 0.990 \\
Low & 6.000 & $1.458-24.686$ & 0.013 & 8.568 & $1.186-61.906$ & 0.033 \\
\hline
\end{tabular}

Adjusted potential covariates for multivariate analysis included age, gender, and BMI; PTC, papillary thyroid carcinoma; OR, odds ratio; $\mathrm{Cl}$, confidence interval;

BMI, body mass index

As shown in Table 5 and Table 6, logistic regression analysis exhibited that PTCs with a high UIC was identified as the independent predictor for the risk of the high SLC34A2 protein expression (univariate analysis: $\mathrm{OR}=8.381,95 \% \mathrm{CI}=1.770$ 39.692, $\mathrm{P}=0.007$; multivariate analysis: $\mathrm{OR}=7.780,95 \%$ $\mathrm{CI}=1.165-37.488, \mathrm{P}=0.011$ ) and the low SLC4A4 protein expression (univariate analysis: $\mathrm{OR}=17.333$, 95\% CI=1.902-157.999, $\mathrm{P}=0.011$; multivariate analysis: $\mathrm{OR}=18.179,95 \% \mathrm{CI}=1.840-179.554, \mathrm{P}=0.013)$. These findings illustrated that a high UIC might be closely linked to the high SLC34A2 and low SLC4A4 protein expression.

Table 5. Logistic regression analysis of high UIC for high SLC34A2 expression risk of PTC patients

\begin{tabular}{lllllll}
\hline UIC, $\mu \mathrm{g} / \mathrm{L}$ & \multicolumn{2}{l}{ Univariate analysis } & \multicolumn{4}{l}{ Multivariate analysis } \\
\cline { 2 - 7 } & OR & $95 \% \mathrm{CI}$ & P value & OR & $95 \% \mathrm{CI}$ & P value \\
\hline $100-299$ & 1.00 & & & 1.00 & & \\
$<100$ & 4.571 & $0.673-31.048$ & 0.120 & 4.405 & $0.641-30.284$ & 0.132 \\
$\geq 300$ & 8.381 & $1.770-39.692$ & 0.007 & 7.780 & $1.165-37.488$ & 0.011 \\
\hline
\end{tabular}

PTC, papillary thyroid carcinoma; OR, odds ratio; CI, confidence interval; UIC, urinary iodine concentration.

Table 6. Logistic regression analysis of high UIC for low SLC4A4 expression risk of PTC patients

\begin{tabular}{|c|c|c|c|c|c|c|}
\hline \multirow[t]{2}{*}{$\mathrm{UIC}, \mu \mathrm{g} / \mathrm{L}$} & \multicolumn{3}{|c|}{ Univariate analysis } & \multicolumn{3}{|c|}{ Multivariate analysis } \\
\hline & OR & $95 \% \mathrm{CI}$ & P value & OR & $95 \% \mathrm{CI}$ & P value \\
\hline $100-299$ & 1.00 & & & 1.00 & & \\
\hline$<100$ & 2.167 & $0.299-15.705$ & 0.444 & 2.338 & $0.292-18.746$ & 0.424 \\
\hline$\geq 300$ & 17.333 & $1.902-157.999$ & 0.011 & 18.179 & $1.840-179.554$ & 0.013 \\
\hline
\end{tabular}

\section{Locating analysis}

The representative staining images obtained from IHC assays are shown in Figure 5. Consistent with the above findings, the positive staining intensity of SLC34A2 protein expression was demonstrated to be markedly enhanced in the cytoplasm of follicular epithelial cells of the tumor tissue of PTCs who suffered with capsular invasion and extra-thyroid metastasis, compared to their corresponding adjacent normal thyroid. Contrastingly, the negative staining intensity of SLC4A4 protein expression was notably weak in the cytomembranes and nuclei of follicular epithelial cells of the tumor tissue of PTCs who suffered with capsular invasion and extra-thyroid metastasis, compared to their corresponding adjacent normal thyroid. These results might help to determine the cellular localization of SLC34A2 and SLC4A4 protein expression level in tumor tissue of PTC patients who have undergone capsular invasion and extra-thyroid metastasis. 


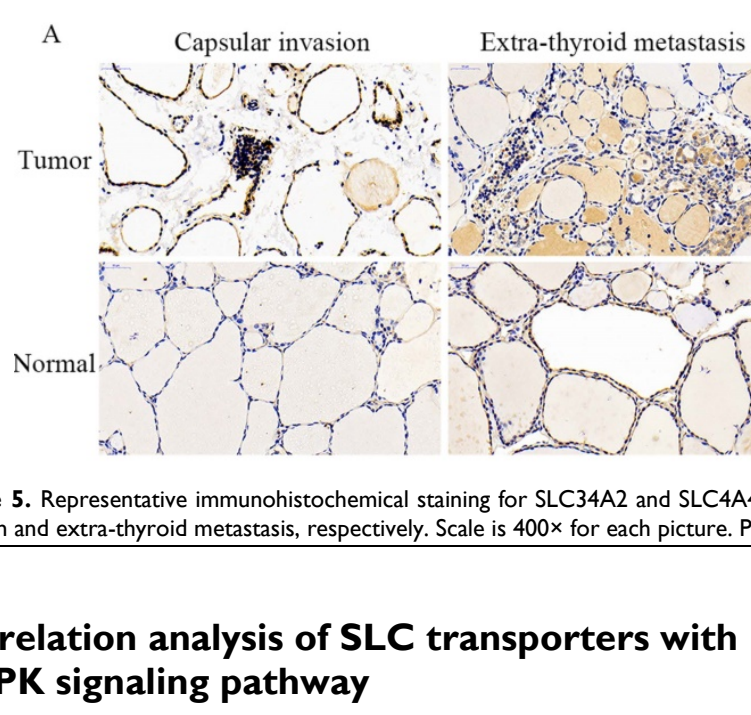

Association between MAPK signaling pathway with capsular invasion and extra-thyroid metastasis

As shown in Figure 6, the results of the western blotting showed that $\mathrm{p}$-ERK protein expression was significantly up-regulated by $17.8 \%$ and $29.2 \%$ for capsular invasion and extra-thyroid metastasis tumors, respectively, when compared to their corresponding adjacent normal thyroid $(\mathrm{P}$ all $<0.05)$. The protein expression of p-JNK was markedly up-regulated by $67.1 \%$ for extra-thyroid metastasis tumors, compared to the adjacent normal thyroid ( $\mathrm{P}<$ 0.05). The protein expression of p-P38 was significantly up-regulated by $38.9 \%$ for extra-thyroid metastasis tumors, when compared to the adjacent normal thyroid $(\mathrm{P}<0.05)$. However, no significant differences were observed for the protein expression of p-ERK, p-JNK, and p-P38 between tumor tissues and their corresponding adjacent normal thyroids ( $P$ all $>0.05$ ) of the PTCs who cannot undergone any capsular invasion nor extra-thyroid metastasis. Hence, the phosphorylation activation of ERK might be both related to tumor capsular invasion and extra-thyroid metastasis of PTCs, while phosphorylation activation of JNK and P38 might be only related to extra-thyroid metastasis.

\section{Correlations between SLC34A2 and SLC4A4 with phosphorylation of ERK, JNK, and P38}

Western blotting was used to determine the possible association between SLC34A2 and SLC4A4 protein expression with the p-ERK, p-JNK, and p-P38 protein expression in tumor tissues when compared to the adjacent normal thyroid tissue. As shown in Figure 7, a significantly negative correlation was observed between SLC4A4 protein expression with $\mathrm{p}-\mathrm{JNK}(\mathrm{r}=-0.696, \mathrm{P}=0.004)$ and $\mathrm{p}-\mathrm{P} 38(\mathrm{r}=-0.534, \mathrm{P}$ $=0.049$ ). Unfortunately, no remarkable correlations were observed between the relative expression of SLC34A2 with p-ERK, p-JNK, and p-P38 (P all > 0.05), as well, between SLC4A4 and p-ERK (P > 0.05). These results enable us to speculate that SLC4A4 down-regulation is closely associated with the activation of the phosphorylation of JNK and P38, yet SLC34A2 is irrelevant to the activation of the MAPK signaling pathway.

\section{Discussion}

Generally, SLC transporters are serve as the transmembrane protein that can facilitate the exchange of various substances and molecules across intracellular and extracellular. Thereby, some SLC transporters were regarded as potential therapeutic targets for certain tumors, such as in the case of the sodium/iodine symporter, the norepinephrine transporter, and the monocarboxylate transporter [21]. Increasing evidence suggests that SLC transporters not only function to transport the endogenous and xenobiotic compounds, but also may play a pivotal role in the initiation and progression of thyroid carcinomas [22, 23]. However, the clinicopathological significance of the SLC transporter expression in patients of PTC, as well as its involvement in the regulation mechanism of signaling pathways have not yet been completely elucidated. In this study, through bioinformatics analysis from the GEO database, and combined with mining results of TCGA clinical cohort database, the gene, namely SLC34A2 (mediate phosphate absorption), SLC4A4 (transport sodium bicarbonate), and SLC25A15 (exchange ornithine and citrulline), were established as the preliminary candidate differentially expressed SLC transporters for further experiment research. And the validation results of RT-PCR experiment demonstrated that the SLC34A2 mRNA was drastically up-regulated while SLC4A4 mRNA was significantly down-regulated; however, SLC25A15 mRNA showed no significant differences between tumor and normal tissue; furthermore, the trends of protein expression were the same as those of mRNA levels. It should be noted that SLC34A2 was markedly overexpressed both in thyroid cancer and gastric 
cancer, whereas SLC4A4 knockdown promote proliferation, migration, and invasion of MDA-MB-231 breast cancer cells and LS174 colon cancer cells [12, 22]. Together, these information
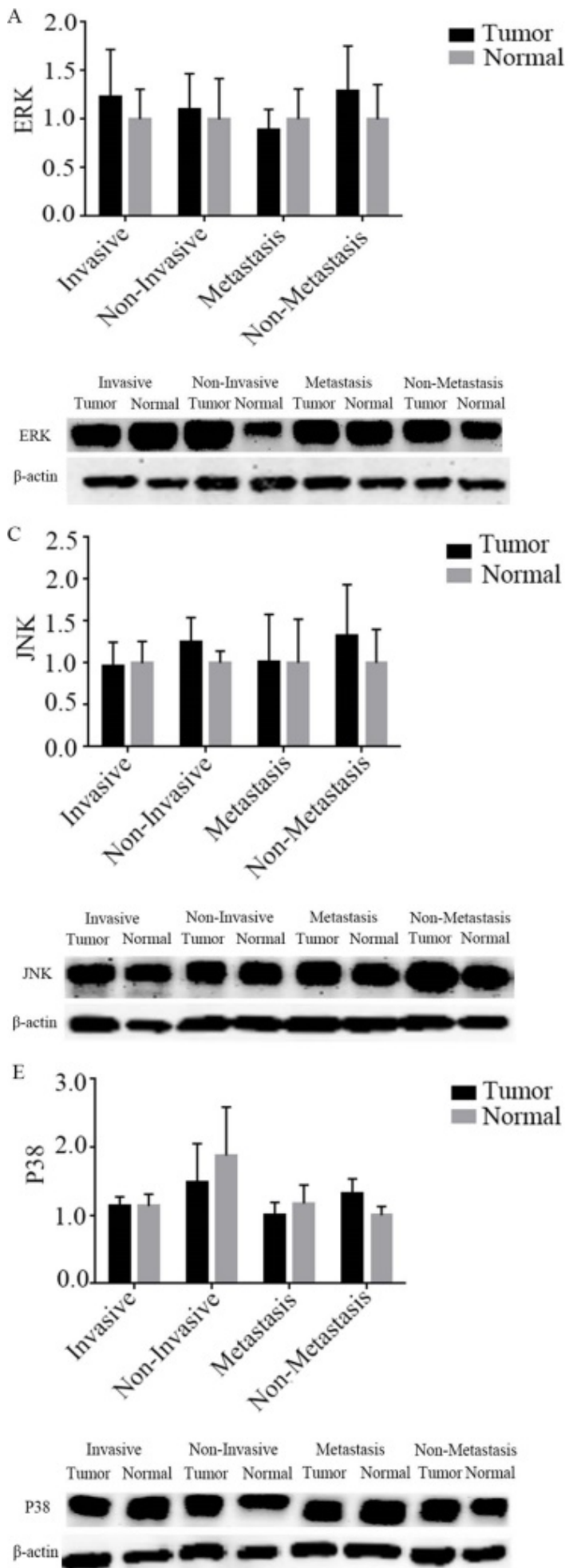

Figure 6. Expression of MAPK signaling pathway in tumor tissues and adjacent normal thyroid of PTCs who suffered with capsule invasion and extra-thyroid metastasis by western blotting. A-F were namely ERK, P-ERK, JNK, P-JNK, P38, and P-P38 “*” represented P < 0.05, “**” represented P < 0.01 . PTC, papillary thyroid carcinoma.

sufficient supported that SLC34A2 and SLC4A4 were the true differentially expressed SLC transporters of PTCs.
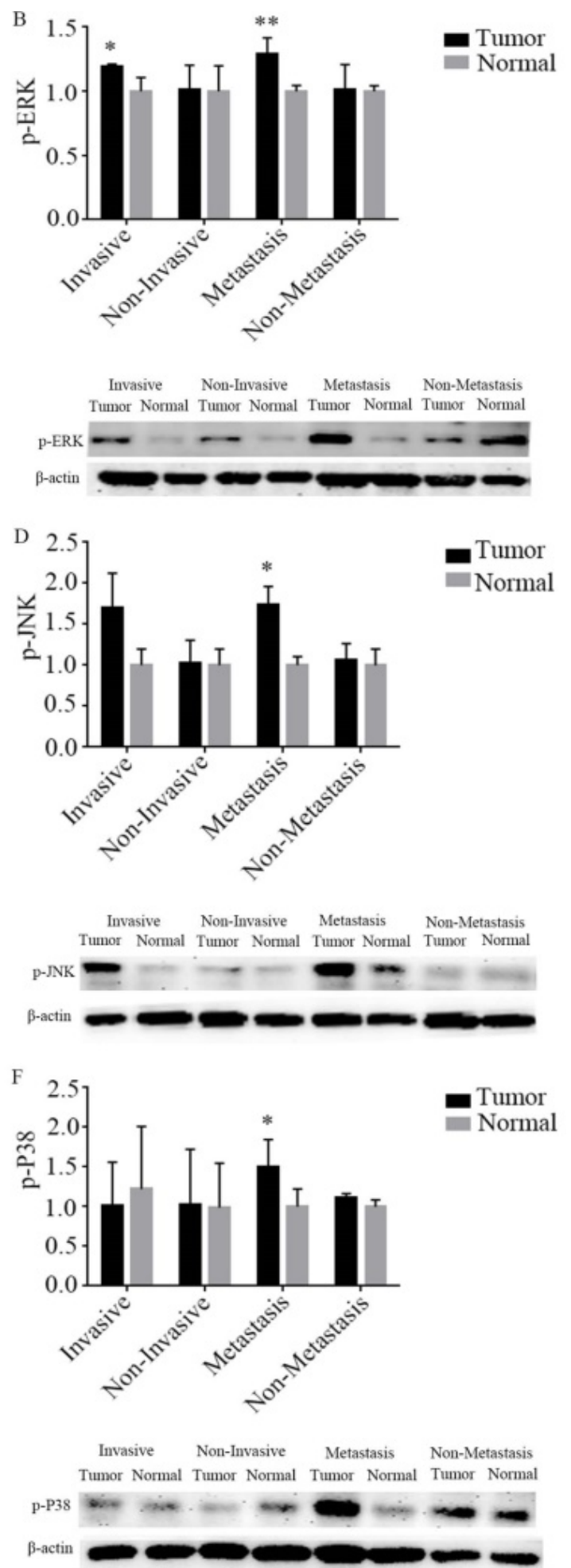

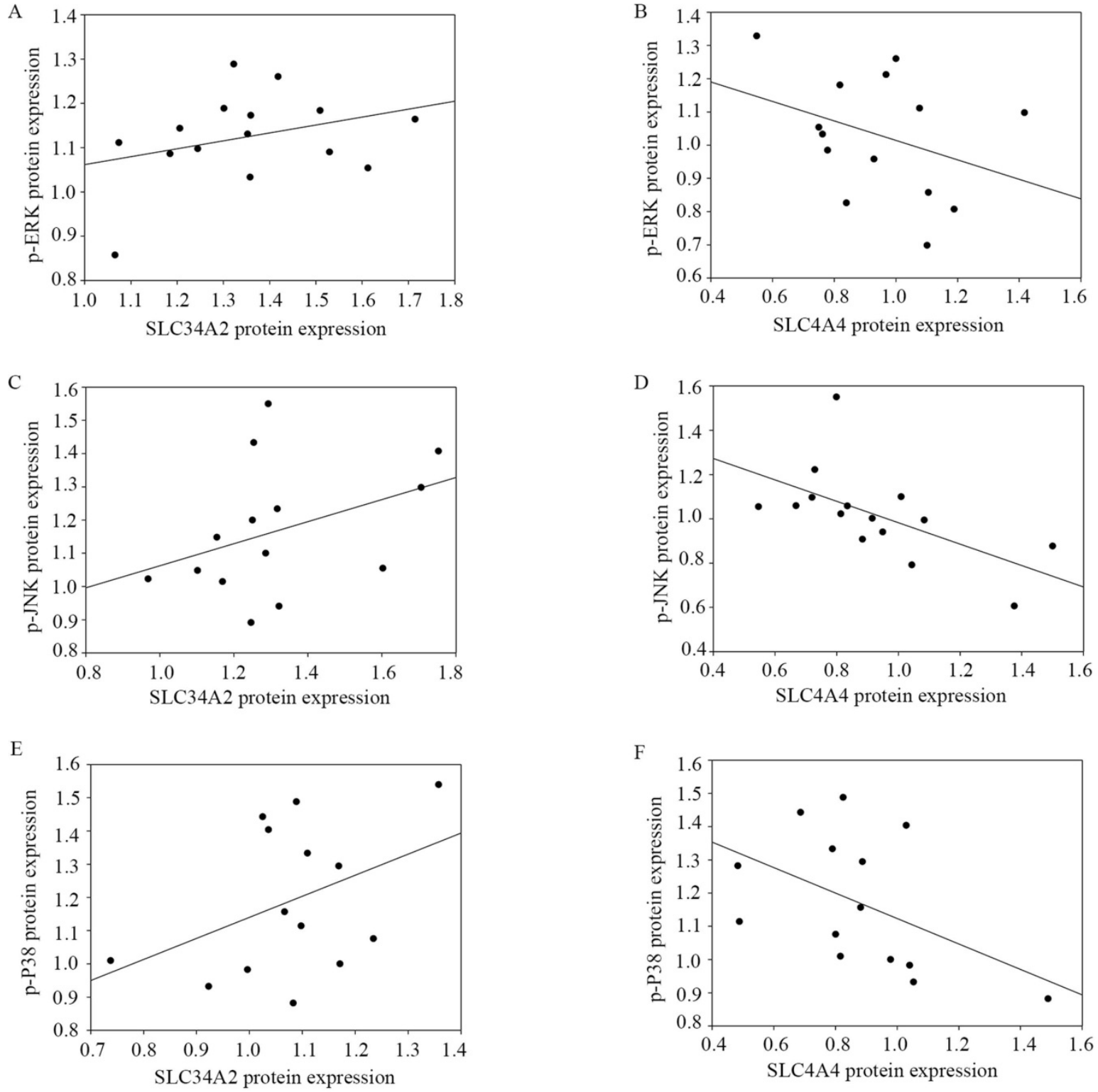

Figure 7. Correlation of SLC34A2 and SLC4A4 expression with MAPK signaling pathway of PTCs. A and $B$ namely the correlation between $p-E R K$ with SLC34A2 ( $r=0.225$, $P=0.420)$ and SLC4A4 $(r=-0.354, P=0.215) ; C$ and $D$ namely the correlation between $P-J N K$ with SLC34A2 $(r=0.437, P=0.118)$ and $S L C 4 A 4(r=-0.696, P=0.004) ; E$ and $F$ namely the correlation between $\mathrm{P}-\mathrm{P} 38$ with SLC34A2 $(r=0.336, \mathrm{P}=0.240)$ and SLC4A4 $(r=-0.534, \mathrm{P}=0.049)$. PTC, papillary thyroid carcinoma.

Notably, a characteristic of this study is the fact that the clinicopathological significance of SLC34A2 and SLC4A2 protein expression for patients with PTC were preliminary evaluated. The results of western blotting revealed that the percentage of capsular invasion and extra-thyroid metastasis were markedly higher in patients with high levels of SLC34A2 $(90.5 \%$ vs $54.5 \%$ and $76.2 \%$ vs $40.9 \%, \mathrm{P}$ all $<0.05)$ and low levels of SLC4A4 (93.7\% vs $54.2 \%$ and $75.0 \%$ vs $33.3 \%$, $\mathrm{P}$ all < 0.05), and high SLC34A2 protein expression can serve as a risk predictor both for capsular invasion and extra-thyroid metastasis while low SLC4A4 protein expression can only serve as a risk predictor for extra-thyroid metastasis. It was reported that the abnormal expression of SLC transporters might be significantly linked to both the local invasion and distant metastases presented in patients with thyroid carcinoma [23, 24]. Thus, it can be inferred that high level of SLC34A2 both closely interrelated capsular invasion risk and extra-thyroid metastasis risk, whereas low SLC4A4 level was only closely related to the risk of extra-thyroid metastasis. Moreover, localization results illustrated that positive staining of the SLC34A2 protein was mainly enhanced in the cytoplasm of thyroid follicular epithelial cells in tumor of PTCs who undergone capsular invasion and extra-thyroid metastasis, while SLC4A4 protein was found to be weak in the cytomembrane and nuclei. These results commonly indicated SLC34A2 protein overexpression might be ascribed for the SLC34A2 expression enhancement in cytoplasm; in contrast, low SLC4A4 expression might be attributed to poor 
SLC4A4 expression both in cytomembrane and nucleus. Taken together, it should be speculated that the enhancement of SLC34A2 protein expression in the cytoplasm might be contribute to the risk of both capsular invasion and extra-thyroid metastasis, whereas the reduction of SLC4A4 protein expression in the cytomembrane and nucleus might be only closely related to the risk of extra-thyroid metastasis.

An incidental discovery of this study is that the expression of both SLC34A2 and SLC4A4 proteins might be disturbed by the individual's excessive iodine nutrition intakes. Comparative results exhibited that the proportion of high levels of UIC ( $\geq$ $300 \mathrm{ug} / \mathrm{L})$ in patients with high SLC34A2 protein expression might be higher than that in PTC patients with unchanged expression $(76.2 \%$ vs $31.8 \%, \mathrm{P}<0.05)$; meanwhile, the proportion of high levels of UIC $(\geq 300$ $\mathrm{ug} / \mathrm{L})$ in patients with low SLC4A4 protein expression was higher than that in patients with unchanged expression $(81.2 \%$ vs $37.5 \%, \mathrm{P}<0.05)$. Logistic regression analysis results also illustrated a high UIC might serves as the independent predictor for the risk of high SLC34A2 and low SLC4A4 of PTC patients. It is also well known that UIC is a widely accepted evaluate indicator of individual's iodine nutritional intakes, because more than $90 \%$ of ingested iodine is excreted through the urine. Furthermore, previous study demonstrated that high iodine stimulation (supplemented dose, $7.3 \mathrm{mg} / \mathrm{L}$ ) can markedly perturb the mRNA and protein expression of SLC transporters (SLC5A5 and SLC26A4) in thyroid tissues of Wistar rats, in comparison to control group [25]. Combined with the results of our previous research that high iodine closely associated with the risk of capsule invasion and thyroid metastasis of PTCs [19]. Therefore, these data together supported a speculation that excess iodine intakes might through up-regulated of SLC34A2 and down-regulated of SLC4A4, contributed to capsule invasion and extra-thyroid metastasis of PTCs. However, further functional experiments needed to verify of this speculation.

With regard to mechanisms, on the one hand, the activation of MAPK signaling pathway has been proven to be the indispensable factor in the occurrence and progression of PTCs [26, 27]. However, on the other hand, previous reports have suggested that the suppression of SLC34A2 can repress tumor cell growth, migration, and metastasis of gastric cancer through inhibition of phosphorylated ERK [28]. Activation of SLC4A4 via inhibited the phosphorylated of P38 to limit the cell death in ischemia-reperfusion injury rat hearts [29]; however, inhibition of JNK (SP600125, $10 \mathrm{uM}$ ) cannot effect SLC4A4 protein abundance in cortical astrocytes cells
[30]. Notably, ERK, JNK, and P38 are together compose the MAPK signaling pathway [31]. Therefore, it can be hypothesized that the MAPK signaling pathway might act as a pivotal downstream (rather than upstream) signaling pathway for SLC34A2 and SLC4A4, to promote the tumor progression of PTCs. Based on this hypothesis, we determined to detect the key molecules of the MAPK signaling pathway by western blotting to explore the possible regulatory mechanisms for SLC34A2 and SLC4A4 that underwent capsular invasion and extra-thyroid metastasis. Our results demonstrated that p-ERK was significantly up-regulated by $17.8 \%$ in tumors for capsular invasion, while p-ERK, p-JNK, and p-P38 were significantly up-regulated by $29.2 \%$, $67.1 \%$, and $38.9 \%$, in tumors that underwent extra-thyroid metastasis, respectively. Thus, the activation of p-ERK might contribute to both capsular invasion and extra-thyroid metastasis; however, the activation of p-JNK and p-P38 might solely responsible for extra-thyroid metastasis. In one aspect, the activation of ERK could via the acceleration of epithelial-mesenchymal transition (EMT) [32] to provoke PTC cells (TPC-1 and K1) invasiveness and migration, which was previously detected by CCK8 assay [33, 34]. However, in other aspect, both P38 (inhibitor, SB203580) and JNK (inhibitor, SP600125) inhibition can generate intracellular reactive oxygen species (ROS) [35], and blocking apoptosis [36] and stimulating stroma-secreted inflammation [37], which could commonly promote the tumor cell metastasis of PTCs $[38,39]$. Therefore, we speculated that p-ERK activation might through accelerate the EMT and contribute to the capsular invasion and extra-thyroid metastasis of PTCs, whereas p-JNK and p-P38 might be activated through the generation of intracellular ROS, blocked apoptosis, and stimulated inflammation, which together contributed to the extra-thyroid metastasis of PTCs.

Furthermore, linear correlation analysis were utilized to evaluate the association between the MAPK signaling pathway with SLC34A2 and SLC4A4. The results showed that only SLC4A4 was significantly negatively correlated with p-JNK $(\mathrm{r}=-0.696, \mathrm{P}<0.05)$ and $\mathrm{p}-\mathrm{P} 38(\mathrm{r}=-0.534, \mathrm{P}<0.05)$. Together with aforementioned mentioned hypothesis that MAPK signaling pathway may serve as a pivotal downstream signal for SLC4A4 to provoke PTC tumor progression. Therefore, it should be inferred that low SLC4A4, via the activation of JNK/P38 MAPK signaling pathway, contribute to the risk of extra-thyroid metastasis of PTCs. However, our results also illustrated that the MAPK signaling pathway does not the significant downstream that 
correlate with SLC34A2. Therefore, we can infer that SLC34A2 contributed to the risk of capsular invasion and extra-thyroid metastasis not via MAPK signaling pathway, that process might involve in other significantly regulatory pathways, such as AKT [40], NF-кB [41], JAK/STAT [42], and Wnt/ $\beta$-Catenin [43].

There are some limitations which should be considered when interpreting the results of this study. First, the conclusion would have been more powerful if multiple centers with larger sample sizes were used as the prospective cohort. Second, this was a preliminary exploration of the distribution of gene expression based on clinical cross-sections with small sample sizes, and present can only aid in drawing the possible correlations between SLC34A2 and SLC4A4 with invasion, metastasis, and excess iodine intake of PTCs; subsequent efforts should involve in a series of in vivo and in vitro gain- or loss-of-function experimental assays (K1 and TPC- 1 , and xenograft tumor in nude mice) [44] to reveal the upstream and downstream and further explore deeper mechanisms. Lastly, refer to the published experiment design (Wang et al. [45] and Liu et al. [46]), considered the advantages of each experiment and the purpose of each step were differences, we only performed western blotting experiment on all of cases and made statistical analysis conclusions, and did not perform RT-PCR and IHC for all 48 cases. Furthermore, our results only supported that SLC34A2 contributed to capsular invasion and extra-thyroid metastasis not via the MAPK signaling pathway, but the possible pathway functioning downstream of SLC34A2 cannot be conclude currently. Despite all these limitations, this study still clarify the clinicopathological significance of differentially expressed SLC transporters and explored possible regulatory signaling pathway which be involved, to some extent. Future work should emphasize in vivo and in vitro gain- or loss-of-function experimental to validate these findings and further clarify which the major signaling pathway is regulated by SLC34A2 in tumor progression of PTCs.

In conclusion, SLC34A2 (mainly in cytoplasm) up-regulated may contribute to the increased risk of both capsular invasion and extra-thyroid metastasis of PTCs, but this effects may not via the MAPK signaling pathway; whereas SLC4A4 (mainly in the cytomembrane and nucleus) down-regulated might contribute to the risk of extra-thyroid metastasis of PTCs, and this effects through the activation of JNK/P38 MAPK signaling pathway. Furthermore, high SLC34A2 and low SLC4A4, might be all attributable to excessive iodine intakes of individuals. Undoubtedly, the results will provide a new perspective for the research of the role of SLC transporters in invasion and metastasis of PTCs, and more beneficial for deeper molecular mechanism exploration, clinical biomarker identification, and novel therapeutic target discoveries.

\section{Abbreviations}

PTC: papillary thyroid carcinoma; SLC: solute carrier; GEO: Gene Expression Omnibus; TCGA: The Cancer Genome Atlas; DEGs: differentially expressed genes; RT-PCR: real-time polymerase chain reaction; ROS: reactive oxygen species; EMT: endothelial-mesenchymal transition; UIC: urinary iodine concentration; IHC: immunohistochemical; TIRADS: Thyroid Imaging Reporting and Data System.

\section{Supplementary Material}

Supplementary tables.

http://www.jcancer.org/v12p5439s1.pdf

\section{Acknowledgements}

The authors would like to thank to the Department of Central Laboratory, Department of Pathology, and Evidence-Based Medicine Center, The Second Hospital of Shandong University.

\section{Funding}

This project was supported by the Research Development Fund of The Second Hospital of Shandong University (grant no.11681808) and Jinan clinical medical science and technology innovation plan (grant no. 202019194).

\section{Author Contributions}

$\mathrm{FH}, \mathrm{JL}, \mathrm{ZY}$, and HJ designed the study. FH, HW, $\mathrm{YZ}, \mathrm{WC}, \mathrm{MG}, \mathrm{JS}, \mathrm{LS}, \mathrm{ZH}$, and LW collected the samples and performed the molecular experiment. FH, JX, CS, and SD analyzed the experimental data. $\mathrm{FH}$ drafted the manuscript, and ZY, JL and HJ made further revisions. All authors read and approved the final version of the manuscript.

\section{Ethics approval and consent to participate}

The present study was approved by the Research Ethics Committee of The Second Hospital of Shandong University; ethics approval no. [KYLL-2019(KJ)P-0084]. All patients were informed of the purpose of the study and volunteered to participate.

\section{Availability of materials and data}

All data generated or analyzed in the present study are available from the corresponding author upon reasonable request. 


\section{Competing Interests}

The authors have declared that no competing interest exists.

\section{References}

1. Sui C, Liang N, Du R, He Q, Zhang D, Li F, et al. Time trend analysis of thyroid cancer surgery in China: single institutional database analysis of 15,000 patients. Endocrine. 2020; 68(3):617-628

2. Abdullah MI, Junit SM, Ng KL, Jayapalan JJ, Karikalan B, Hashim OH. Papillary Thyroid Cancer: Genetic Alterations and Molecular Biomarker Investigations. Int J Med Sci. 2019; 16: 450-460.

3. Tan J, Qian X, Song B, An X, Cai T, Zuo Z, et al. Integrated bioinformatics analysis reveals that the expression of cathepsin $S$ is associated with lymph node metastasis and poor prognosis in papillary thyroid cancer. Oncol Rep. 2018; 40: 111-122.

4. Bensimon A, Pizzagalli MD, Kartnig F, Dvorak V, Essletzbichler P, Winter GE, et al. Targeted Degradation of SLC Transporters Reveals Amenability of Multi-Pass Transmembrane Proteins to Ligand-Induced Proteolysis. Cell Chem Biol. 2020; 27(6):728-739.

5. Morioka S, Perry JSA, Raymond MH, Medina CB, Zhu Y, Zhao L, et al. Efferocytosis induces a novel SLC program to promote glucose uptake and lactate release. Nature. 2018; 563: 714-718.

6. Hu C, Tao L, Cao X, Chen L. The solute carrier transporters and the brain: Physiological and pharmacological implications. Asian J Pharm Sci. 2020; 15: 131-144.

7. Brecht K, Schäfer AM, Meyer Zu Schwabedissen HE. Uptake Transporters of the SLC21, SLC22A, and SLC15A Families in Anticancer Therapy-Modulators of Cellular Entry or Pharmacokinetics? Cancers (Basel). 2020; 12(8):2263.

8. Lai B, Lai Y, Zhang Y, Zhou M, Sheng L, OuYang G. The Solute Carrier Family 2 Genes Are Potential Prognostic Biomarkers in Acute Myeloid Leukemia. Technol Cancer Res Treat. 2020; 19: 1533033819894308.

9. Kikuchi D, Saito M, Saito K, Watanabe Y, Matsumoto Y, Kanke Y, et al. Upregulated solute carrier family 37 member 1 in colorectal cancer is associated with poor patient outcome and metastasis. Oncol Lett. 2018; 15: 2065-2072.

10. Ding B, Lou W, Xu L, Li R, Fan W. Analysis the prognostic values of solute carrier (SLC) family 39 genes in gastric cancer. Am J Transl Res. 2019; 11(1):486-498.

11. El-Ansari R, Craze ML, Alfarsi L, Soria D, Diez-Rodriguez M, Nolan CC, et al. The combined expression of solute carriers is associated with a poor prognosis in highly proliferative ER+ breast cancer. Breast Cancer Res Treat. 2019; 175(1):27-38.

12. He J, Zhou M, Li X, Gu S, Cao Y, Xing T, et al. SLC34A2 simultaneously promotes papillary thyroid carcinoma growth and invasion through distinct mechanisms. Oncogene. 2020; 39(13):2658-2675.

13. Horvath E, Majlis S, Rossi R, Franco C, Niedmann JP, Castro A, et al. An ultrasonogram reporting system for thyroid nodules stratifying cancer risk for clinical management. J Clin Endocrinol Metab. 2009; 94(5):1748-1751.

14. [No authors listed]. The TCGA Legacy. Cell. 2018; 173(2):281-282.

15. Livak KJ, Schmittgen TD. Analysis of relative gene expression data using real-time quantitative PCR and the 2(-Delta Delta $\mathrm{C}(\mathrm{T}))$ Method. Methods. 2001; 25(4):402-408.

16. Bradford MM. A rapid and sensitive method for the quantitation of microgram quantities of protein utilizing the principle of protein-dye binding. Anal Biochem. 1976; 72: 248-254.

17. Huang $\mathrm{F}, \mathrm{Wu} \mathrm{Y}$, Zhang D, Liu X, Wang Z. Carbon disulfide induced decidualization disorder in the mice uterus at the window of implantation. Ecotoxicol Environ Saf. 2020; 190: 110069

18. Huang F, Sun Y, Gao H, Wu H, Wang Z. Carbon disulfide induces embryo loss by perturbing the expression of the mTOR signalling pathway in uterine tissue in mice. Chem Biol Interact. 2019; 300: 8-17.

19. Huang F, Cong W, Xiao J, Zhou Y, Gong M, Sun J, et al. Association between excessive chronic iodine exposure and the occurrence of papillary thyroid carcinoma. Oncol Lett. 2020; 20(5):189.

20. Yuan J, Song Y, Pan W, Li Y, Xu Y, Xie M, et al. LncRNA SLC26A4-AS1 suppresses the MRN complex-mediated DNA repair signaling and thyroid cancer metastasis by destabilizing DDX5. Oncogene. 2020; 39(43):6664-6676.

21. Zhang Y, Wang J. Targeting uptake transporters for cancer imaging and treatment. Acta Pharm Sin B. 2020; 10(1):79-90.

22. Scott KP, Jacques P. The $\mathrm{Na}(+) / \mathrm{HCO} 3(-)$ Co-Transporter SLC4A4 Plays a Role in Growth and Migration of Colon and Breast Cancer Cells. J Cell Physiol. 2015; 230(8):1954-1963.
23. Lan L, Deng W, Chen HL, Huo LL, Deng LL, Zhang GY, et al. [All-trans retinoic acid improves iodine uptake of thyroid cancer cells via repressing transcriptional activity of $\beta$-catenin]. Zhonghua Yi Xue Za Zhi. 2016; 96(7):553-558.

24. Tavares C, Coelho MJ, Eloy C, Melo M, da Rocha AG, Pestana A, et al. NIS expression in thyroid tumors, relation with prognosis clinicopathological and molecular features. Endocr Connect. 2018; 7(1): 78-90.

25. Serrano-Nascimento C, Salgueiro RB, Vitzel KF, Pantaleão T, Corrêa da Costa VM, Nunes MT. Iodine excess exposure during pregnancy and lactation impairs maternal thyroid function in rats. Endocr Connect. 2017; 6(7):510-521.

26. Yu ST, Zhong Q, Chen RH, Han P, Li SB, Zhang H, et al. CRLF1 promotes malignant phenotypes of papillary thyroid carcinoma by activating the MAPK/ERK and PI3K/AKT pathways. Cell Death Dis. 2018; 9(3):371.

27. Wang DP, Tang XZ, Liang QK, Zeng XJ, Yang JB, Xu J. Overexpression of long noncoding RNA SLC26A4-AS1 inhibits the epithelial-mesenchymal transition via the MAPK pathway in papillary thyroid carcinoma. J Cell Physiol. 2020; 235(3):2403-2413.

28. Zhang JX, Xu Y, Gao Y, Chen C, Zheng ZS, Yun M, et al. Decreased expression of miR-939 contributes to chemoresistance and metastasis of gastric cancer via dysregulation of SLC34A2 and Raf/MEK/ERK pathway. Mol Cancer. 2017; 16(1):18.

29. Ciocci Pardo A, González Arbeláez LF, Fantinelli JC, Aiello EA, Mosca SM. Calcineurin/P38MAPK/HSP27-dependent pathways are involved in the attenuation of postischemic mitochondrial injury afforded by sodium bicarbonate co-transporter (NBCe1) inhibition. Biochem Pharmacol. 2019; 161: 26-36.

30. Khakipoor S, Ophoven C, Schrödl-Häußel M, Feuerstein M, Heimrich B, Deitmer JW, et al. TGF- $\beta$ signaling directly regulates transcription and functional expression of the electrogenic sodium bicarbonate cotransporter 1, NBCe1 (SLC4A4), via Smad4 in mouse astrocytes. Glia. 2017; 65(8):1361-1375.

31. Lanna A, Gomes DC, Muller-Durovic B, McDonnell T, Escors D, Gilroy DW, et al. A sestrin-dependent Erk-Jnk-p38 MAPK activation complex inhibits immunity during aging. Nat Immunol. 2017; 18(3): 354-363.

32. Lin X, Zhang H, Dai J, Zhang W, Zhang J, Xue G, et al. TFF3 Contributes to Epithelial-Mesenchymal Transition (EMT) in Papillary Thyroid Carcinoma Cells via the MAPK/ERK Signaling Pathway. J Cancer. 2018; 9(23):4430-4439.

33. Zhou T, Zhong M, Zhang S, Wang Z, Xie R, Xiong C, et al. LncRNA CASC2 expression is down- regulated in papillary thyroid cancer and promotes cell invasion by affecting EMT pathway. Cancer Biomark. 2018; 23(2):185-191.

34. Gao Y, Elamin E, Zhou R, Yan H, Liu S, Hu S, et al. FKBP51 promotes migration and invasion of papillary thyroid carcinoma through NF-kB-dependent epithelial-to-mesenchymal transition. Oncol Lett. 2018; 16(6):7020-7028

35. Song TH, Chen XX, Lee CK, Sze SC, Feng YB, Yang ZJ, et al. Dendrobine targeting JNK stress signaling to sensitize chemotoxicity of cisplatin against non-small cell lung cancer cells in vitro and in vivo. Phytomedicine. 2019; 53: 18-27.

36. Ahn J, Chung YW, Park JB, Yang KM. $\omega$-hydroxyundec-9-enoic acid induces apoptosis by ROS mediated JNK and p38 phosphorylation in breast cancer cell lines. J Cell Biochem. 2018; 119(1):998-1007.

37. Meng J, Wang DM, Luo LL. CTRP3 acts as a novel regulator in depressive-like behavior associated inflammation and apoptosis by meditating p38 and JNK MAPK signaling. Biomed Pharmacother. 2019; 120: 109489.

38. Parascandolo A, Rappa F, Cappello F, Kim J, Cantu DA, Chen H, et al. Extracellular Superoxide Dismutase Expression in Papillary Thyroid Cancer Mesenchymal Stem/Stromal Cells Modulates Cancer Cell Growth and Migration. Sci Rep. 2017; 7: 41416.

39. Liu TR, Su X, Qiu WS, Chen WC, Men QQ, Zou L, et al. Thyroid-stimulating hormone receptor affects metastasis and prognosis in papillary thyroid carcinoma. Eur Rev Med Pharmacol Sci. 2016; 20(17):3582-3591.

40. Liu X, Zhou X, Xu H, He Z, Shi X, Wu S. SLC34A2 Regulates the Proliferation, Migration, and Invasion of Human Osteosarcoma Cells Through PTEN/PI3K/AKT Signaling. DNA Cell Biol. 2017; 36(9):775-780

41. Lin $\mathrm{Y}, \mathrm{Ma} \mathrm{Q}, \mathrm{Li} \mathrm{L}$, Wang $\mathrm{H}$. The CXCL12-CXCR4 axis promotes migration, invasiveness, and EMT in human papillary thyroid carcinoma B-CPAP cells via NF-kB signaling. Biochem Cell Biol. 2018; 96(5):619-626.

42. Bi CL, Zhang YQ, Li B, Guo M, Fu YL. MicroRNA-520a-3p suppresses epithelial-mesenchymal transition, invasion, and migration of papillary thyroid carcinoma cells via the JAK1-mediated JAK/STAT signaling pathway. J Cell Physiol. 2019; 234(4):4054-4067. 
43. Chen LL, Gao GX, Shen FX, Chen X, Gong XH, Wu WJ. SDC4 Gene Silencing Favors Human Papillary Thyroid Carcinoma Cell Apoptosis and Inhibits Epithelial Mesenchymal Transition via Wnt/ $\beta$-Catenin Pathway. Mol Cells. 2018; 41(9):853-867.

44. Ding Z, Ke R, Zhang Y, Fan Y, Fan J. FOXE1 inhibits cell proliferation, migration and invasion of papillary thyroid cancer by regulating PDGFA. Mol Cell Endocrinol. 2019; 493: 110420.

45. Wang L, Zhou W, Zhong Y, Huo Y, Fan P, Zhan S, et al. Overexpression of $G$ protein-coupled receptor GPR87 promotes pancreatic cancer aggressiveness and activates NF-KB signaling pathway. Mol Cancer. 2017; 16(1):61.

46. Liu L, Yang Y, Zhou X, Yan X, Wu Z. Solute carrier family 34 member 2 overexpression contributes to tumor growth and poor patient survival in colorectal cancer. Biomed Pharmacother. 2018; 99: 645-654. 